\title{
Have You Tested Your Goldmann Tonometer Today?
}

\author{
Nikhil S Choudhari, Ronnie George, L Vijaya
}

Medical Research Foundation, Sankara Nethralaya, 18, College Road, Chennai (India)

\section{INTRODUCTION}

Intraocular pressure (IOP) is the only known modifiable risk factor for glaucoma. Besides being a cornerstone in glaucoma management, accurate measurement of IOP forms an important component of routine ophthalmic evaluation. Despite the innovation of various newer tonometers, Goldmann applanation tonometer is still considered the Gold standard for IOP measurement. However, there are several reported sources of error including calibration error. ${ }^{1}$

Errors in calibration of Goldmann applanation tonometer are often overlooked in clinical practice. Kumar and Jivan ${ }^{2}$ conducted a questionnaire survey of 100 ophthalmology residents from UK. 85\% of respondents to their questionnaire never checked tonometers for errors. ${ }^{2}$ Wessels and $\mathrm{Oh}^{3}$ did a field survey of 94 ophthalmologists' offices to assess the accuracy of tonometers in daily use. Less than 15\% of ophthalmologists in their study knew how to perform calibration error testing. ${ }^{3}$ Given the importance of applanation tonometry in glaucoma management and the ease of calibration error testing, these figures are surprising.

\section{METHOD OF TESTING THE CALIBRATION ERROR OF GOLDMANN APPLANATION TONOMETER}

The calibration error of Goldmann applanation tonometer can be measured at 3 testing levels, viz. 0, 2 and 6 corresponding to 0,20 and $60 \mathrm{mmHg}$ of IOP respectively. Of these 3 levels, the level corresponding to $20 \mathrm{mmHg}$ is the most important, as many clinical decisions with regard to glaucoma are taken around this IOP. Haag-Streit, the largest maker of the Goldmann tonometer, also mentions this as the most important testing level. ${ }^{4}$

The manufacturers of Haag-Streit Goldmann applanation tonometer provide a calibration error check weight bar (Fig. 1). ${ }^{4}$ The bar has 5 markings on it. The central marking corresponds to level 0 . Two on either side of it represent level 2 and the two outermost markings represent level 6.

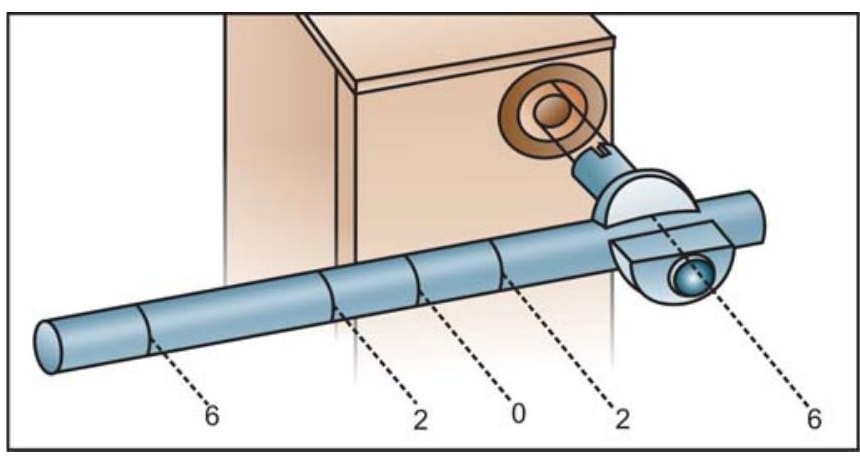

Fig. 1: Goldmann applanation tonometer calibration error check weight bar provided by the Haag-Streit company

The method of testing calibration error of the Goldmann applanation tonometers has been described in detail by the manufacturer. ${ }^{4} \mathrm{~A}$ video presentation in the current issue of this journal also describes the procedure.

There are two basic models of the Goldmann applanation tonometer. The 900 series mounts on the Haag-Streit or similar type slit lamp. The 870 series mounts on the tower top of a Zeiss or similar type slit lamp. The calibration error check weight bar and its holder fit into the slot provided on the side of the 900 model (Fig. 2) or at the junction of the balance arm of the 870 model of the Goldmann applanation tonometer (Fig. 3). After setting the mark on the weight bar corresponding to one of the three testing positions on the index mark of the weight holder, the revolving knob of the measuring drum should be rotated forwards. The reading at which the feeler arm with the applanation prism in place moves forward freely should be recorded. The difference of this reading from the actual test position ( 0,2 or 6 corresponding to 0,20 and $60 \mathrm{~mm} \mathrm{Hg}$ of IOP) indicates the positive error at that level of testing in $\mathrm{mm} \mathrm{Hg}$. Similarly, on rotating the revolving knob in the reverse direction, the reading at which the feeler arm moves backward should be 


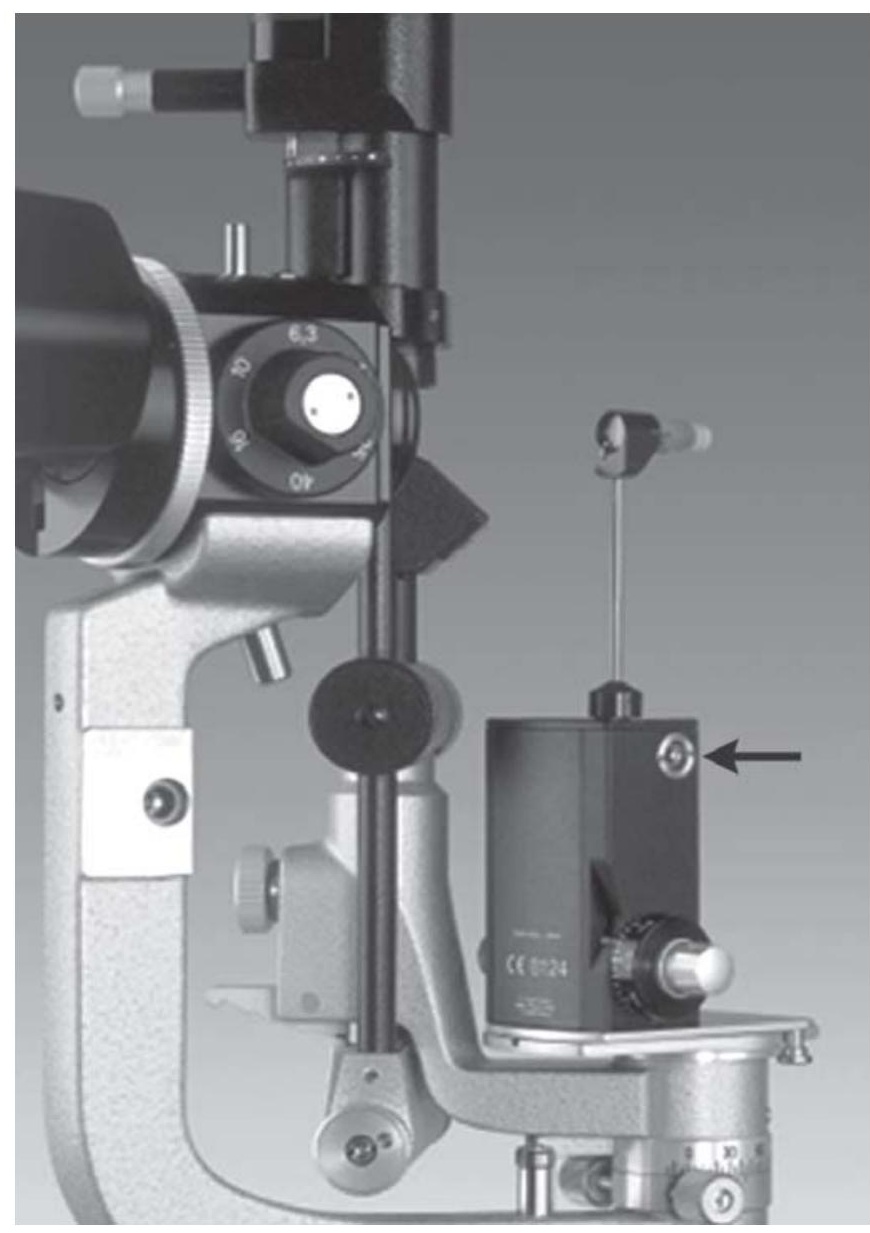

Fig. 2: Goldmann applanation tonometer of 900 series attached to a slitlamp. The arrow indicates the slot to fit the holder of the calibration error check weight bar

noted. The difference between the latter and the testing position, indicates the negative error at that level of testing. Calibration error testing at level 'zero' can be performed even without the calibration error check weight bar.

While testing calibration error, one must be attentive. It is easy not to tighten the calibration error check bar at the precisely correct position, especially when changing the settings in a hurry, and get an erroneous calibration error. It is also to be noted that the calibration error check bar should be positioned with the long arm towards the examiner while changing the settings, since the Goldmann applanation tonometer is based on a balancing principle.

One must note that, by this method one can only measure the amount of calibration error in a particular Goldmann applanation tonometer at that particular time. The manufacturers of Haag-Streit Goldmann applanation tonometer recommend that any tonometer with an unacceptable calibration error should be returned to them for rectification of calibration error. ${ }^{4}$

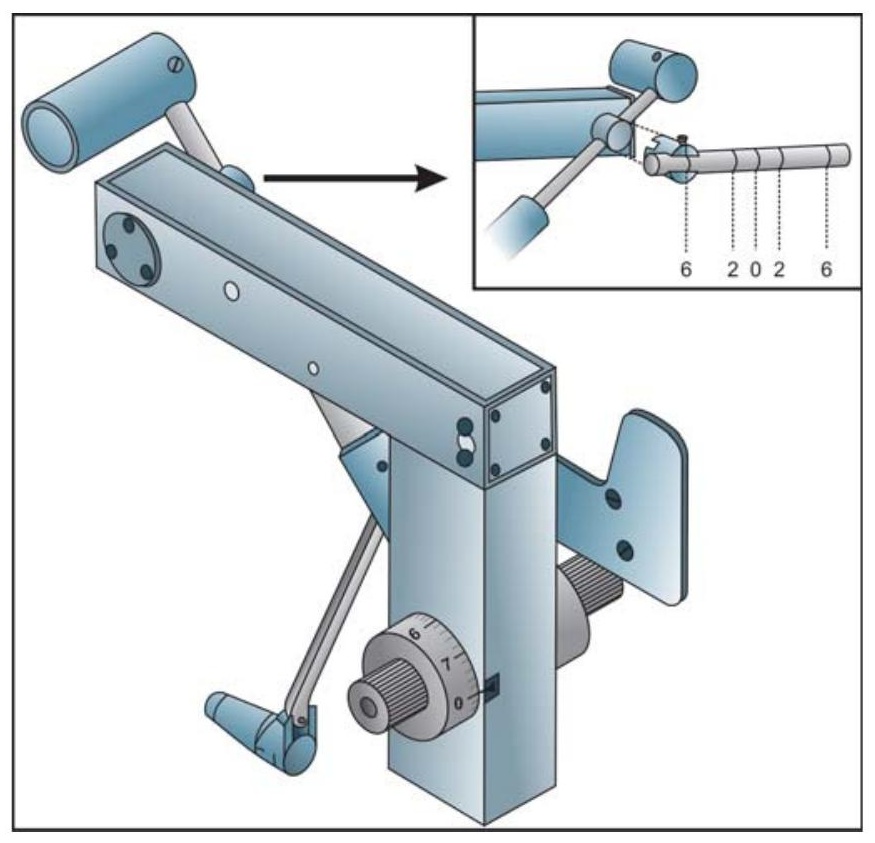

Fig. 3: Goldmann applanation tonometer of 870 series attached to a slitlamp. The arrow indicates the magnified image of the slot to fit the holder of the calibration error check weight bar

\section{AGREEMENT IN THE MEASUREMENT OF CALIBRATION ERROR OF GOLDMANN APPLANATION TONOMETER}

The intraobserver and interobserver agreement in the measurement of calibration error of Goldmann applanation tonometer is rarely reported in the literature. Ray-Chaudhury and Birch in their reply to the editor ${ }^{5}$ have reported the interobserver agreement in the measurement of calibration errors of Goldman applanation tonometer. However, they only rate the agreement as 'good', but do not mention the method of agreement used or the actual figures.

We studied the intraobserver and interobserver agreement in the measurement of calibration error of Goldmann applanation tonometer. We found only a moderate Intra Class Correlation (ICC) for intraobserver agreement in the measurement of positive calibration error. However, the intraobserver agreement for negative error as well as the interobserver agreement for both positive and negative errors by ICC was good in our study. ${ }^{6}$ The 95\% limits of intra and inter observer agreement, by the Bland and Altman method, ${ }^{7}$ in the measurement of negative calibration error were narrower than the corresponding limits of intra- and inter-observer agreement in the measurement of positive calibration error in our study. ${ }^{6}$ The reason as well as the significance for this observation is unclear. Traditionally, while measuring IOP, we 'ascend', and rarely 'descend', from the mark ' 1 ' on the measuring drum of the Goldmann applanation tonometer. Therefore, intra- and inter- observer agreement in the measurement of positive calibration error should carry more 
importance. We also looked at the actual difference in the number of Goldmann tonometers that were classified as acceptable or faulty while calculating intra- or inter-observer agreement. This difference was minimal in our study. ${ }^{6}$

\section{DEFINING CALIBRATION ERROR TOLERANCE FOR GOLDMANN APPLANATION TONOMETER}

The manufacturers of Haag-Streit Goldmann applanation tonometer define acceptable calibration error to be within \pm 0.5 $\mathrm{mm} \mathrm{Hg}$ at all levels of testing (0, 20 and $60 \mathrm{~mm} \mathrm{Hg})$. On the other hand, the South-East Asia Glaucoma Interest Group (SEAGIG) guideline is less stringent and recommend that the acceptable range of calibration error should progressively widen at the higher levels of error testing. ${ }^{8}$ By this guideline, the acceptable error can be within $\pm 2 \mathrm{~mm} \mathrm{Hg}$ at $0 \mathrm{~mm} \mathrm{Hg}, \pm 3 \mathrm{~mm} \mathrm{Hg}$ at $20 \mathrm{~mm}$ $\mathrm{Hg}$ and $\pm 4 \mathrm{~mm} \mathrm{Hg}$ at $60 \mathrm{~mm} \mathrm{Hg}$ testing levels. ${ }^{8}$

The range of calibration error for the Goldmann applanation tonometer can potentially influence patient care. If glaucomatous damage is advanced it could adversely influence clinical decision making. Calibration errors may not cause such clinically significant errors in early or moderate disease.. In clinical practice, patients with varying severity of glaucoma are seen. One needs to strike a balance between a very strict calibration criterion that is practically difficult to achieve versus a lax standard that leads to errors in management.

\section{FREQUENCY OF CALIBRATION ERROR OF GOLDMANN APPLANATION TONOMETER}

Goldmann applanation tonometers that are heavily used have reportedly much higher frequency of calibration errors compared to the ones that are sparingly used. Over the 4-month period of their study, ${ }^{9}$ Sandhu, Chattopadhyay, Birch et al. found only 0 to $10.3 \%$ of Goldmann tonometers to be within the manufacturer's recommended error tolerance. On applying the same criterion, only 5 (4\%) of our Goldmann tonometers were acceptable at any level of testing. ${ }^{6}$ Both these studies ${ }^{6,9}$ were performed on heavily used Goldmann applanation tonometers. In contrast, Wessels and $\mathrm{Oh}^{3}$ reported 104 (82\%) while Chuo and Mikelberg ${ }^{10}$ reported 17 (68\%) slit lamp mounted Goldmann tonometers within the manufacturer's recommended calibration range. However, Wessels and Oh's study ${ }^{3}$ was based on a field survey of solo practitioners. Those tonometers were not subjected to heavy daily usage and multiple users. Chuo and Mikelberg $^{10}$ did not record data on frequency of use of the tonometers.

\section{MAGNITUDE OF CALIBRATION ERROR OF GOLDMANN APPLANATION TONOMETER}

The reported range of calibration error of Goldmann applanation tonometer is variable. In our study, ${ }^{6}$ the range of calibration error was -8 to $+20 \mathrm{~mm} \mathrm{Hg}$. In contrast, Wessels and $\mathrm{Oh}^{3}$ reported a range of only -2.5 to $+3.5 \mathrm{mmHg}$. In all studies done so far, ${ }^{3,6,9,10}$ tonometers were more likely to show a positive calibration error than the negative error. This may lead to an over-estimation of true IOP. While the impact of this overestimation of IOP is difficult to quantify, it could be clinically significant. It can also lead to over diagnosis of glaucoma or over treatment in individual patients.

\section{FREQUENCY OF CALIBRATION ERROR TESTING FOR GOLDMANN APPLANATION TONOMETER}

There is no practice guideline in the literature regarding the frequency of calibration error testing. The manufacturer suggests an arbitrary monthly check. ${ }^{4}$ Some authors recommend an arbitrary annual check ${ }^{1,3}$ and others ${ }^{9}$ suggest an arbitrary monthly check.

In our study, ${ }^{6}$ the tonometers initially found faulty were repaired and subsequently released for use. Four months later, $11(20.75 \%)$ of the 53 initially faulty Goldmann tonometers were found to have drifted out of calibration. On this basis, at least 4 monthly formal calibration error testing with the use of the calibration error check weight bar is advisable.

At $0 \mathrm{mmHg}$, calibration error testing can be performed easily without use of the calibration error check weight bar. ${ }^{4}$ In our study, ${ }^{6} 67.5 \%$ of Goldmann tonometers that were faulty at $20 \mathrm{~mm} \mathrm{Hg}$ were also faulty at $0 \mathrm{mmHg} .{ }^{6}$ It is simple to screen Goldmann applanation tonometers at $0 \mathrm{mmHg}$ without using the calibration error check weight bar. The screening could even be performed daily, especially in busy ophthalmic practices. One may involve optometrists or even paramedical staff for this simple but important task.

Seventy percent of ophthalmology residents from UK felt that calibration testing was not part of their responsibility. ${ }^{2}$ Knowing this, Kumar and Hillier, in their national survey, ${ }^{11}$ assessed the views of senior nursing staff in charge of running ophthalmology outpatient clinics as to whom they feel to be responsible for Goldmann tonometer calibration error checks. A significant number of nursing staff believed calibration checks were not their responsibility. This lack of communication between health care professionals is alarming and can lead to the use of inaccurate tonometers in clinical practice. These observations suggest that ophthalmic practices should create a fixed protocol that clearly identifies individuals responsible for checking Goldmann applanation tonometer calibration error at a predetermined frequency.

\section{SOURCES OF CALIBRATION ERROR OF GOLDMANN APPLANATION TONOMETER}

Sources of calibration error of Goldmann applanation tonometer are rarely investigated. As a part of the study protocol, ${ }^{6}$ our bioengineers repaired 11 faulty instruments on the spot. The calibration error of a majority of these instruments (9 out of 11) was corrected after cleaning and lubrication of component parts alone. 
The Goldmann applanation tonometer modifies force by adjusting a spring. In a properly functioning instrument, according to the Hook's law, the deformation produced in the spring, i.e. strain should be directly proportional to the deforming IOP, i.e. stress within elastic limit. We should investigate the reasons why Hook's law does not remain linear in Goldmann tonometers with calibration errors. Accumulation of dirt and loss of adequate lubrication with repeated use of the instrument may be some of them.

The reported frequency of calibration errors of Goldmann applanation tonometer in our study ${ }^{6}$ and in the study by Sandhu, Chattopadhyay, Birch et $\mathrm{al}^{9}$ is higher than that reported by Wessels and $\mathrm{Oh}^{3}$ or Chuo and Mikelberg. ${ }^{10}$ Heavy use of the instruments and multiple users turn out to be the common factors between our study ${ }^{6}$ and that by Sandhu Chattopadhyay and Birch et al. ${ }^{9}$ These two factors may explain the difference in the observations. These observations also indicate that Goldmann tonometers can rapidly develop calibration errors in routine clinical use, emphasizing the need for frequent calibration error checks to ensure accuracy.

Older Goldmann applanation tonometers may have a higher frequency of calibration errors due to a wear and tear process. In our study, ${ }^{6}$ no instruments that were less than 2 years old were faulty. In contrast, high frequencies of older instruments were faulty. Wessels and $\mathrm{Oh}^{3}$ found a statistically significant negative correlation between the age of the Goldmann tonometer and accuracy.

One specific cause for calibration error is accidental damage. The Goldmann applanation tonometers are of two basic typesbase-plate tonometers and slit-lamp mounted tonometers. Baseplate tonometers may be more prone for accidental damage. Wessels and $\mathrm{Oh}^{3}$ found a statistically significant difference in calibration errors between mounted and hand-held tonometers (19\% versus 33\%, p 0.02). Sandhu, Chattopadhyay, Birch et al ${ }^{9}$ also studied this aspect but could not demonstrate a difference in the frequency of calibration errors between the two subtypes of the Goldmann tonometer. However, the numbers studied by them are small. Any tonometer that suffers damage should undergo a calibration error check before continuing to be used.

Since the Goldmann applanation tonometer is a balancing instrument, any tilt in the surface on which the slit lamp assembly is mounted can possibly cause errors in measurement of IOP.

\section{RECTIFYING CALIBRATION ERROR OF GOLDMANN APPLANATION TONOMETER}

The manufacturer's acceptable range of calibration error tolerance is very narrow. Moreover, the manufacturer recommends faulty tonometers should be returned to them for calibration. However, to the best of our knowledge, there is no repair facility in India and the logistics and time delay do not permit such an arrangement in most ophthalmic practices in the country. A significant number of faulty instruments in our study ${ }^{6}$ needed lubrication of component parts alone. Lubrication of the instruments if not repair, can be done by bioengineers or technicians of any institute and may drastically cut down the number of instruments that need to be sent to the manufacturer.

The issue of repair of Goldmann applanation tonometer needs to be resolved. Special training of existing bioengineers or technicians at various eye care facilities by the manufacturer is one of the possible solutions.

\section{REFERENCES}

1. Whitacre MM, Stein R. Sources of error with use of Goldmanntype tonometers. Surv Ophthalmol 1993;38:1-30.

2. Kumar N, Jivan S. Goldmann applanation tonometer calibration error checks: Current practice in the UK. Eye. 2007;21(6):733-34.

3. Wessels IF, Oh Y. Tonometer utilization, accuracy, and calibration under field conditions. Arch Ophthalmol 1990;108:1709-12.

4. Haag-Streit Intl. Goldmann AT 900/870. Applanation Tonometer to slit lamp. Procedures for checking tonometer calibration. 2008:1-4. Available at: http://haag-streit-usa.com/pdf/ procedures-for-checking-tonometer calibration.pdf. Accessed June 11, 2008.

5. Ray-Chaudhury N, Birch M. In Reply of: Wessels IF. Frequency of Goldmann applanation tonometer calibration error checks [letter]. J Glaucoma 2006;15:74-75.

6. Choudhari NS, George R, Baskaran M, Vijaya L, Dudeja N. Measurement of Goldmann Applanation Tonometer Calibration Error. Accepted for publication in Ophthalmology [in press].

7. Bland JM, Altman DG. Statistical Methods for Assessing Agreement Between Two Methods of Clinical Measurement. Lancet 1986;I:307-10.

8. South-East Asia Glaucoma Interest Group. Asia Pacific Glaucoma Guidelines. 2003-04:76. Available at: http://www.seagig.org/toc/ APGGuidelinesNMview. pdf. Accessed June 11, 2008.

9. Sandhu SS, Chattopadhyay S, Birch MK, Ray-Chaudhuri N. Frequency of Goldmann applanation tonometer calibration error checks. J Glaucoma 2005;14:215-18.

10. Chuo JY, Mikelberg FS. Calibration errors of Goldmann tonometers in a tertiary eye care centre. Can J Ophthalmol 2007;42:712-14.

11. Kumar N, Hillier RJ. Goldmann tonometer calibration: A national survey. Eye. 2007 Oct 26. [Epub ahead of print]

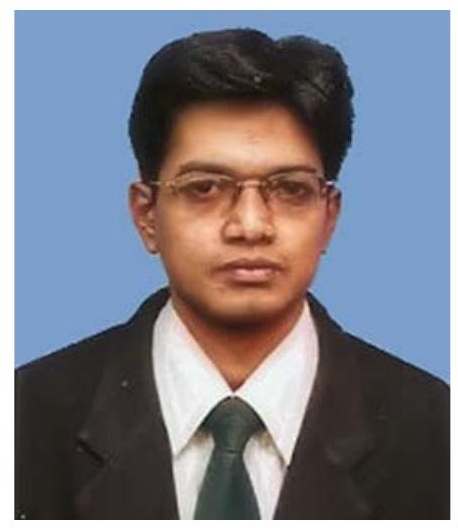

Nikhil S Choudhari

(drnkl@snmail.org) 